Research Journal of Medical Sciences 6 (3): 148-153, 2012

ISSN: $1815-9346$

(C) Medwell Journals, 2012

\title{
Quran Recitation: Short-Term Effects and Related Factors in Preterm Newborns
}

\author{
${ }^{1}$ Narges Eskandari, ${ }^{2}$ Maryam Keshavars, ${ }^{3}$ Hassan Ashayeri, \\ ${ }^{2}$ Fereshte Jahdi and ${ }^{4}$ Agha Fatemeh Hosseini \\ ${ }^{1}$ Department of $\mathrm{Ob}$ and Gyn, School of Nursing Midwifery, Khalkhal Branch, \\ Islamic Azad University, Khalkhal, Iran \\ ${ }^{2}$ Department of $\mathrm{Ob}$ and Gyn, School of Nursing Midwifery, \\ ${ }^{3}$ Department of Rehabilitation Sciences, \\ ${ }^{4}$ Department of Biostatistics, School of Management and Medical Information, \\ Tehran University of Medical Sciences, Tehran, Iran
}

\begin{abstract}
The effects of Holy Quran recitation on anxiety and vital signs in adults have been reported in several studies in Iran but have not been studied in preterm newborns. In a randomized controlled trial, 120 preterm newborns in a teaching hospital were randomly allocated to either a control or experimental group. Quran recitation by Shahhat Mohammad Anvar played via headphones with a volume range of 50-60 db in the experimental group. The two groups were surveyed for short term physiological responses at minute 10 and 20 of intervention also minute 10 post intervention. Between two groups, statistically significant differences were observed in respiratory rate and oxygen saturation during three time points but difference in heart rate was seen only at minute 10 post intervention $(\mathrm{p}=0.012)$. All three variables in experimental group were significantly different in three time points $(\mathrm{p}<0.0001)$. Gender, gestational age and neonate weight were factors that decreased respiratory rate. Holy Quran recitation can be considered a complementary and supportive care for preterm newborns whereas gender, gestational age and neonate weight were related factors in response to the Holy Quran recitation.
\end{abstract}

$\underline{\text { Key words: Quran recitation, physiological responses, preterm newborn, heart rate, respiratory, Iran }}$

\section{INTRODUCTION}

Sound therapy has been described as a non-invasive and alternative health approach to wellness (Meyer et al., 2009 ) and music alone or combined with the human voice seems to be a valuable resource to provide developmental stimulations in preterm newborns to reduce stress, promote bonding with parents and enhance developmental goals in the Neonatal Intensive Care Unit (NICU) (Standley, 2002). Preterm births have increased since 1990 with a $30 \%$ increase over the past 25 years. Of the 4 million babies born in the USA each year, about $500,000(12.5 \%)$ arrive prematurely (Tramo et al., 2009). In Iran, $13.5 \%$ of neonates were born preterm in 2003 (ONIC, 2007).

Many preterm infants suffer from neurological disabilities including cerebral palsy, visual and hearing impairments; learning difficulties and psychological, behavioural and social problems (Neal, 2008). The negative effects of stress, pain, adverse environmental stimuli and isolation from parents may be reflected in variations in heart rate, decrease in oxygen saturation level, wide fluctuations in blood pressure and increased agitation (Courtnage, 2001). The increasing incidence of prematurity, prevalence of significant morbidity and burden to society, make it imperative to identify developmental care strategies and health patterning modalities such as music that might reduce this burden (Neal, 2008). Thus, studies on premature infants in the NICU have primarily focused on medical procedures for survival and complementary techniques (Standley, 2001).

Because auditory capability and discrimination develop so early, several studies have focused on the effects of auditory stimuli on preterm infants (Standley, 2000). Standley (2002) in a meta-analysis suggested that music has clinically important benefits for preterm infants including increased oxygen saturation, reduced stress, promotion of bonding with family members, reinforcement of non-nutritive sucking, increased sucking ability and sustained homeostasis during multimodal stimulation (Standley, 2002). Positive effects on long-term variables have been reported including length of hospitalization

Corresponding Author: Maryam Keshavarz, School of Nursing and Midwifery, Tehran University of Medical Sciences, Tohid Sq., West Nosrat St. Tehran, Iran 
(Cevasco, 2008), weight gain (Schwartz, 1997; Caine, 1991), non-nutritive sucking rate (Standley, 2000) and short term responses such as oxygen saturation (Chou et al., 2003; Cassidy and Standby, 1995), heart rate (Coleman et al., 1998) and behavioural scores (Chapman, 1979; Schwartz, 1997). Chou et al. (2003) reported that music therapy during endotracheal suction, significantly increases oxygen saturation in preterm infants (Chou et al., 2003). In principle, there are persuasive theories and preliminary small studies that support music and singing as appropriate developmental care for preterm infants in the NICU (Neal, 2008).

One of the most complementary methods is music and chant. A melodious chant such as the playing of the Quran, the holy book of Islam is one of the most beautiful. Quran verses are full of internal harmony. The words are shaped with such a unique order of letters so when we hear it, researchers would have been fully impressed by its miraculous harmony and rhythm whether we are familiar with its meaning or not (Marefat, 1993).

In Iran, the effects of Quran recitation on anxiety have been surveyed and vital signs in adult patients have been assessed. These studies reported that Quran recitation can be used as a non-pharmacological and safe procedure to reduce anxiety and to improve vital signs (Majidi, 2004; Abadi et al., 2003; Khatoni, 1997). Researchers conducted this study to investigate the physiological responses of preterm newborns to Quran recitation.

\section{MATERIALS AND METHODS}

Design: A double blind randomized controlled trial was conducted to evaluate the effects of a recorded selected part of the Quran recitation to preterm newborns in the NICU. All preterm newborns were randomly allocated to either the experimental or control groups. The sample size to achieve a power of 0.8 , alpha level of 0.05 and the smallest effect size of 3.5 for physiological responses was 60 per group which was determined in consultation with a biostatistician.

Settings and subjects: This study was carried out in the NICU of Akbar Abadi Teaching Birth centre as a referring setting. This centre delivers more preterm newborns than any other birth centre in Tehran. Participants were 120 preterm newborns who were hospitalized in the NICU and who were referred to the intermediate section of the NICU (INICU).

Subjects had weights of 1500-2750 g, gestational age 30-37 weeks, Apgar scores $\geq 7$ at 1 and $5 \mathrm{~min}$, they were appropriate for gestational age and had confirmed hearing by the startle reflex. None of the study participants had been undergoing mechanical ventilation, photo therapy, sedative medications such as phenobarbital and did not have intra ventricular haemorrhage, necrotizing enterocolitis, sepsis, acute lung diseases, congenital defects, neonatal anaemia, acute illness and reported hyper-responsiveness to the sound and history of maternal drug abuse including alcohol or illegal substances. Only newborns who were clinically and physically stable were included.

Infant's responses: Short-term physiological responses including oxygen saturation, respiratory rate and heart rate were chosen as dependent variables because they are responsive to auditory stimuli and have been commonly used in studies on the effects of music in preterm newborns.

Experimental intervention: The intervention consisted of Yusuf chapter, verses 7-23 by Shahhat Mohammad Anvar for $20 \mathrm{~min}$ to experimental group via headphones on the morning shift. The volume range was $50-60 \mathrm{db}$ in consultation with a neuroscience specialist and was set with the A-scale of a Cirrus (cr274) sound level meter.

Procedure: This study protocol was approved by the ethics committee of nursing and midwifery school, Tehran University of Medical Sciences and was conducted during August-September 2009. Eligible parents were approached while they were visiting their newborns in the NICU so the purpose and procedure of the study was briefly described to them by the researchers. Parents were encouraged to ask any questions that they had regarding the research then formal written consent was obtained.

Demographic data of newborns were obtained from hospital records then they were screened for normal hearing by a neonatologist and the startle reflex to sound stimuli was confirmed. Before handling any of the newborns, researchers reviewed their medical chart to ensure that there were no changes in the infant's medical condition which would be contraindicated for intervention. We also verbally conferred with the nursing staff to ensure that there was no need for nurses to handle the neonates during the $40 \mathrm{~min}$ the researcher needed to conduct the experiment.

The newborn was placed in a supine position and A4TECH Model EST1987 headphones which were attached to a MP3 player were placed over both ears using a stockinet. The headphones presented the 
stimulus at the same carefully controlled decibel level, attenuated ambient noise allowed for minimal volume, guaranteed binaural presentation of the stimulus and prevented an effect on other newborns in the INICU. Oxygen saturation level was measured with a continuous display on a Zimense Model SC6002XL monitor which was attached by sensors to the anterior and posterior of the foot and shielded from light by carbon paper. All newborns were monitored for heart and respiration rate with the same medical device using three adhesive leads applied in a triangular pattern on the chest. Headphones without sound were used for newborns in the control group.

For each group, the study protocol and measurement of variables were started $30 \mathrm{~min}$ after the last feeding, change of diapers or other routine nursing care. The newborn was placed in a crib, the equipment was set up and base line data recording was obtained for $10 \mathrm{~min}$ after placing the earphones and monitor. For the experimental group, the Quran recitation was started and played for $20 \mathrm{~min}$. During four periods including immediately before, at minutes 10 and 20 of intervention and at minute 10 post-intervention. All physiological data were collected by a co-worker who was blinded to the group assignments.

Interventions and treatments were avoided during the course of the study. If newborns experienced any significant medical problems or handling during intervention and within $10 \mathrm{~min}$ after, they were excluded from the study. In the experimental group, three newborns were excluded from the study due to refusing participation by parents, touching and crying. In the control group, two newborns were excluded for crying and vomiting. None of them had signs of hyper sensitivity to sound such as hiccups, facial grimacing, finger splaying, clenched fingers, heart rate $>200$ or oxygen saturation $<80 \%$.

Limitations of data collection methods: The use of earphones to control sound conditions served to reduce the impact of variations in room sound levels or unplanned environmental sounds that might be considered limitations of this study.

Data analysis: SPSS-PC Version 16 (SPPS Inc., Chicago, IL, USA) was used for the data analysis. Descriptive

statistics were used to describe the sample characteristics. The Kolmogorov-Smirnov test was used to assess the normality of the data distribution. The t-test, $\chi^{2}$-test and Mann-Whitney $U$ and Wilcoxon tests were used for parametric and non-parametric data, respectively. Repeated-measures and Friedman's tests were used to compare the means of data from different time points in the experimental group. A $p<0.05$ was considered significant for all statistical tests. Linear regression was used to assess the effects of related factors on early responses of newborns to the Quran recitation.

\section{RESULTS AND DISCUSSION}

Of the 125 preterm infants, 120 finished the study. Random allocation provided equivalent distributions of variables in the two groups (Table 1). No statistically significant differences were observed for mean oxygen saturation or respiratory and heart rates before the intervention however, significant differences in mean oxygen saturation levels and respiratory rate during the three periods of the study were observed in the experimental group. In addition, heart rate at the minute 10 of post intervention (Table 2). Repeated measures for respiratory and heart rate and Freidman's test for oxygen saturation revealed significant differences in the experimental group during the three periods of study (Table 3).

Only respiratory rate at minute 10 of postintervention was significantly affected by gestational age $(\mathrm{p}=0.015, \beta=-1.91)$, weight at birth $(\mathrm{p}=0.025, \beta=-0.009)$ and time of intervention ( $\mathrm{p}=0.018, \beta=-0.01)$. The t-test results showed that respiratory rate at minutes 10 $(\mathrm{p}=0.049)$ and $20(\mathrm{p}=0.01)$ of intervention and heart rate

Table 1: Demographic characteristics of the samples

\begin{tabular}{|c|c|c|c|}
\hline Characteristics & $\begin{array}{l}\text { Intervention group } \\
(\mathrm{n}=60)\end{array}$ & $\begin{array}{l}\text { Control group } \\
(\mathrm{n}=60)\end{array}$ & $\mathrm{p}$-value \\
\hline$\overline{\mathrm{CS} / \mathrm{NVD}^{\dagger}}$ & $24 / 36$ & $32 / 28$ & 0.143 \\
\hline Female/Male & $29 / 31$ & $29 / 31$ & 1.000 \\
\hline Birth weight (g) & $2233 \pm 362.39^{*}$ & $2194.33 \pm 378.07$ & 0.345 \\
\hline Current weight (g) & $2180.66 \pm 362.26$ & $2158.33 \pm 387.47$ & 0.517 \\
\hline Gestational age (weeks) & $34.1 \pm 1.84$ & $33.75 \pm 0.91$ & 0.431 \\
\hline Age at study (days) & $3.96 \pm 3.44$ & $4.01 \pm 3.06$ & 0.487 \\
\hline Apgar score (1 min) & $8.45 \pm 0.96$ & $8.35 \pm 0.65$ & 0.321 \\
\hline Apgar score $(5 \mathrm{~min})$ & $9.53 \pm 0.59$ & $9.4 \pm 0.61$ & 0.201 \\
\hline
\end{tabular}

Table 2: Physiological responses in Quran recitation and control group

\begin{tabular}{|c|c|c|c|c|c|c|c|c|c|}
\hline \multirow[b]{2}{*}{ Variables } & \multicolumn{3}{|l|}{$10 \mathrm{~min}$} & \multicolumn{3}{|l|}{$20 \mathrm{~min}$} & \multicolumn{3}{|l|}{$30 \min ^{\dagger}$} \\
\hline & Intervention & Control & $\mathrm{p}$-value & Intervention & Control & $\mathrm{p}$-value & Intervention & Control & $\mathrm{p}$-value \\
\hline Oxygen saturation (\%) & $96.03 \pm 3.850^{*}$ & $94.78 \pm 4.040$ & 0.05 & $97.11 \pm 3.080$ & $94.96 \pm 4.120$ & 0.02 & $97.13 \pm 3.620$ & $95.43 \pm 3.830$ & 0.01 \\
\hline Respiratory rate $(\mathrm{N})^{t}$ & $44.76 \pm 12.16$ & $49.85 \pm 10.87$ & 0.01 & $42.03 \pm 12.44$ & $49.48 \pm 11.34$ & 0.00 & $38.93 \pm 11.32$ & $48.66 \pm 10.95$ & 0.00 \\
\hline Heart rate $(N)$ & $130.28 \pm 17.63$ & $131.11 \pm 17.26$ & 0.79 & $126.28 \pm 17.53$ & $130.71 \pm 15.93$ & 0.15 & $124.48 \pm 18.27$ & $132.41 \pm 15.49$ & 0.01 \\
\hline
\end{tabular}

${ }^{*}$ Mean $\pm \mathrm{SD} ;{ }^{\dagger} 10$ th $\mathrm{min}$ of post intervention; ${ }^{\mathrm{t}} \mathrm{No}$. in $1 \mathrm{~min}$ 
Res. J.Med. Sci., 6 (3): 148-153, 2012

Table 3: Effect of Quran recitation on physiological responses in 60 preterm infants during study ${ }^{\#}$

\begin{tabular}{lcrrrr}
\hline Variables & \multicolumn{1}{c}{0 min } & $10 \min$ & $20 \min$ & $30 \mathrm{~min}^{\dagger}$ & $\mathrm{p}$-value \\
\hline Oxygen saturation $(\%)$ & $95.51 \pm 4.500^{*}$ & $96.03 \pm 3.850$ & $97.11 \pm 3.080$ & $97.13 \pm 3.620$ & $\mathrm{p}<0.0001$ \\
Respiratory rate $(\mathrm{N})^{t}$ & $47.76 \pm 12.80$ & $44.76 \pm 12.16$ & $42.03 \pm 12.44$ & $38.93 \pm 11.32$ & $\mathrm{p}<0.0001$ \\
Heart rate $(\mathrm{N})$ & $131.96 \pm 17.85$ & $130.28 \pm 17.63$ & $126.28 \pm 17.53$ & $124.48 \pm 18.27$ & $\mathrm{p}<0.0001$ \\
\hline
\end{tabular}

"Based on repeated measures; "mean $\pm \mathrm{SD}$; ${ }^{\dagger} 10$ th min of post intervention; 'No. in $1 \mathrm{~min}$

Table 4: Comparing of phy siological responses between two sexes

\begin{tabular}{|c|c|c|c|c|c|c|}
\hline \multirow[b]{2}{*}{ Variables } & \multicolumn{2}{|c|}{ Oxygen saturation (\%) } & \multicolumn{2}{|l|}{ Heart rate $(\mathrm{N})^{t}$} & \multicolumn{2}{|c|}{ Respiratory rate $(\mathrm{N})$} \\
\hline & $20 \mathrm{~min}$ & $30 \min ^{\dagger}$ & $20 \mathrm{~min}$ & $30 \min ^{\dagger}$ & $20 \mathrm{~min}$ & $30 \min ^{\dagger}$ \\
\hline Female (29) & $96.17 \pm 3.73^{*}$ & $96.40 \pm 3.54$ & $131.64 \pm 8.04$ & $130.58 \pm 17.04$ & $45.96 \pm 13.20$ & $41.70 \pm 11.93$ \\
\hline Male (31) & $95.89 \pm 3.85$ & $96.15 \pm 3.65$ & $120.55 \pm 15.26$ & $126.17 \pm 17.49$ & $37.82 \pm 10.19$ & $35.96 \pm 10.0$ \\
\hline $\mathrm{p}$-value & 0.765 & 0.506 & 0.013 & 0.131 & 0.01 & 0.049 \\
\hline
\end{tabular}

${ }^{*}$ Mean $\pm \mathrm{SD} ;{ }^{\dagger} 10$ th $\min$ of post intervention; ${ }^{\mathfrak{t}} \mathrm{No}$. in $1 \mathrm{~min}$

at the minute $20(\mathrm{p}=0.013)$ in male neonates was significantly lower than those of female neonates (Table 4).

In the current study, a 20 min Quran recitation in preterm newborns resulted in a significant decrease in heart and respiratory rates and an increase in oxygen saturation level as short-term physiological responses. At minutes 10 and 20 of intervention and at minute 10 postintervention, the respiratory rate was lower and oxygen saturation was higher in the experimental group than those in the control group whereas no significant differences in mean heart rate at minutes 10 and 20 of intervention were observed except at minute 10 post-intervention.

The results of this study are comparable to the effects of Quran recitation in adults and music in newborns which are very controversial. Reports related to the oxygen saturation level were an increase during $20 \mathrm{~min}$ of lullaby (Coleman et al., 1998; Chou et al., 2003), a decrease (Johnston et al., 2007) and no difference during music intervention (Collabra et al., 2003; Neal, 2008). None of these studies reported a significant difference in oxygen saturation level after music intervention. In the current study, a higher level of oxygen saturation in the experimental group indicated a positive effect of the Quran recitation during and post-intervention. A decreased respiratory rate at minutes 10 and 20 of intervention also $10 \mathrm{~min}$ post intervention were the other positive results in the current study. Coleman et al. (1998) reported an increased respiratory rate during $20 \mathrm{~min}$ of lullaby intervention (Coleman et al., 1998) whereas Calabro found no effect of music (Collabra et al., 2003).

No significant difference in heart rate was observed between the two groups at minutes 10 and 20 of intervention which is comparable with the results of two other studies (Collabra et al., 2003; Arnon et al., 2006). Decrease (Coleman et al., 1998) and increase (Elander and Hellstrom, 1995) in heart rate during music intervention are two others. Arnon et al. (2006) who applied live music achieved a lower heart rate after intervention therefore, the finding at minute 10 post intervention can be supported by this report (Arnon et al., 2006).

In two Iranian studies, the effects of Quran recitation before surgery in adult patients were reported as a decrease in State-Trait Anxiety score (Majidi, 2004; Khatoni, 1997) and improvement in vital signs such as blood pressure, respiratory and heart rate (Abadi et al., 2003). These findings support the positive effects of Quran recitation in adults. Improvement in short-term physiological responses in preterm newborns was the positive effect in the current study so that Quran recitation in adult and newborns can be considered in future studies.

Factors that affect the physiological responses to Quran recitation as an auditory stimulation were another part of the study. However, researchers found few related studies. Only one study described a relationship between neonatal weight and physiological responses (Wood, 2008). Auditory functioning, demonstrated by cortical auditory evoked responses in the cerebral cortex and brain-stem is apparent by $26-28$ weeks of gestation so older preterm infants can respond to and process auditory stimulation (Neal, 2008). The results emphasize that the increase in gestational age and birth weight induced the decrease in respiratory rate in pre-term newborns.

Researchers found a significant decrease in respiratory and heart rate in male neonates of the experimental group compared to that in females. Although, differences between physiological responses to music have not been identified in male and female preterm newborns, a study of 40 preterm infants (Standley, 1998) reported that female neonates in the experimental group left the hospital earlier than that of control females (an average of 11.9 days). Lullaby had little effect on male neonates in the experimental group because they left the hospital only 1.5 days earlier than that of control males (Standley, 1998). 


\section{CONCLUSION}

The results of this study showed that Quran recitation may be used as complementary and supportive care because it improved the physiological responses of preterm newborns including oxygen saturation level and respiratory and heart rate. Quran recitation had no unwanted effects on the tested variables and did not interfere with technical aspects or routine care. Thus, this type of recitation can be surveyed for safety and effectiveness in other trials, to apply in the NICU, particularly in Islamic countries.

\section{RECOMMENDATIONS}

Extending the length of follow-up $>10 \mathrm{~min}$ is recommended to identify whether the effect was sustained so we could validate the findings. Further research is needed to assess the feasibility and reproducibility of the results and the long-term effects on the development of behavioural responses in preterm newborns.

\section{ACKNOWLEDGEMENTS}

The researchers thank the staff of the NICU at Shahid Akbar Abadi Teaching Centre for their support. We also deeply appreciate the parents and their neonates who participated in this study. This study was derived from a Master Thesis of Tehran University of Medical Sciences, Nursing and Midwifery School. All sources of funding were provided by Tehran University of Medical Sciences.

\section{REFERENCES}

Abadi, E.I., A.R.S. Moqaddam and S.R. Mazloom, 2003. The effect of holy Quranrecitation on the patient's vital signs before open heart surgery. J. Sabzevar Sch. Med. Sci., 10: 52-58.

Arnon, S., A. Shapsa, L. Forman, R. Regev, S. Bauer, I. Litmanovitz and T. Dolfin, 2006. Live music is beneficial to preterm infants in the neonatal intensive care unit environment. Birth, 33: 131-136.

Caine, J., 1991. The effect of music on the selected stress behaviors, weight, caloric formula intake and length of hospital stay of premature and low birth weight neonates in a newborn intensive care unit. J. Music Ther., 28: 180-192.

Cassidy, J.W. and J.M. Standby, 1995. The effect of music listening on physiological responses of premature infants in the NICU. J. Music Ther., 32: 208-227.
Cevasco, A.M., 2008. The effects of mothers singing on full-term and preterm infants and maternal. J. Music Ther., 45: 273-306.

Chapman, J.S., 1979. Influence of Varied Stimuli on Development of Motor Patterns in the Premature Infant. In: Newborn Behavioral Organization: Nursing Research and Implications, Anderson, G. and B. Raff (Eds.). Alan Liss, New York, USA., Pages: 6180.

Chou, L.L., R.H. Wang, S.J. Chen, 2003. Effect of music therapy on oxygen saturation in premature infants receiving endotracheal suctioning. J. Nurs. Res., 11: 209-216.

Coleman, J., R. Pratt, R. Stoddath, D. Gerstmann and H. Abel, 1998. The effects of male and female singing and speaking voices on selected physiological and behavioral measures of premature infants in the intensive care unit. Int. J. Arts. Medi., 5: 4-11.

Collabra, J.A., R.O. Wolfe and H.E. ShoeMarks, 2003. The effect of recorded sedative music on physiology and behavior of premature infants with respiratory disorders. Austr. J. Music Ther., 14: 3-19.

Courtnage, A., 2001. The effect of live infant directed singing of the heart rate, oxygen saturation level and respiration rate of premature infants in the neonatal intensive care unit. Master Thesis, Hahnemann University, Philadelphia.

Elander, G. and G. Hellstrom, 1995. Reduction of noise levels in intensive care units for infants: Evaluation of an intervention program. Heart Lung, 24: 376-379.

Johnston, C.C., F. Filion and A.M. Nuyt, 2007. Recorded maternal voice for preterm neonates undergoing heel lance. Adv. Neonatal. Care, 7: 258-266.

Khatoni, A.R., 1997. Effect of holy Quran recitation on rate of worry in patients hospitalized in ICU. University of Medical Sciences, Iran.

Majidi, S.A., 2004. Recitation effect of Holy Quran on anxiety of patients before undergoing coroner artery angiography. J. Gilan Univ. Med. Sci., 49: 61-67.

Marefat, M.H., 1993. Rhythm in Quran. Mirase Javidan, 1: $10-21$.

Meyer, W.L., G. Neff and L. Garfield-O'Brien, 2009. A summary of sound therapy and vibrational healing concepts. http://www.healingsounds.com.

Neal, D.O., 2008. Music as a health patterning modality for preterm infants in the NICU. Ph.D. Thesis, Minnesota University, USA.

ONIC, 2007. Incidence of preterm labor and low birth weight neonates and effective factors in Iran. Ministry of Health and Medical Education, Health Department, Tehran. 
Schwartz, F.J., 1997. Perinatal stress reduction, music and medical cost savings. J. Prenat. Perinat. Psychol. Health, 12: 19-29.

Standley, J.M., 1998. The effect of music and multimodal stimulation on physiological and developmental responses of premature infants in neonatal intensive care. J. Pediatr. Nurs., 24: 532-539.

Standley, J.M., 2000. The effect of contingent music to increase non-nutritive sucking of premature infants. J. Pediatr. Nurs., 26: 493-499.

Standley, J.M., 2001. Music therapy for the neonate. Newborn Infant Nurs Rev., 1: 211-216.
Standley, J.M., 2002. A Meta-analysis of the efficacy of music therapy for premature infants. J. Pediatr. Nurs., 17: 107-113.

Tramo, M., C. Koh, M. Lense, C. Ness, K. Krishnamoorthy and J. Kagan, 2009. Effect of auditory stimulation with vocal music on neurophysiological responses to acute pain in premature infants. The Institute for Music and Brain Science, 2006, http:/www.BrainMusic.org. Accessed 2009.

Wood, A.H., 2008. Effect of music therapy on premature infant in the neonatal intensive care unit. Seoul, Korea J. Music Ther., 45: 165-191. 